\title{
Synuclein: A Neuron-Specific Protein Localized to the Nucleus and Presynaptic Nerve Terminal
}

\author{
Luc Maroteaux, James T. Campanelli, and Richard H. Scheller \\ Department of Biological Sciences, Stanford University, Stanford, California 94305
}

\begin{abstract}
We used an antiserum against purified cholinergic synaptic vesicles from Torpedo and expression screening to isolate a cDNA clone encoding synuclein, a 143 amino acid neuronspecific protein. A cDNA clone was also isolated from a rat brain cDNA library that encodes a highly homologous 140 amino acid protein. The amino terminal 100 amino acids of both proteins are comprised of an 11 amino acid repeating unit that contains a conserved core of 6 residues. The synuclein gene is expressed only in nervous system tissue, not in electric organ, muscle, liver, spleen, heart, or kidney. In the electric organ synapse Torpedo synuclein-immunoreactive proteins are found in 3 major molecular-weight classes of $17.5,18.5$, and $20.0 \mathrm{kDa}$. In the neuronal cell soma the $17.5 \mathrm{kDa}$ species is predominant and immunoreactivity is localized to a portion of the nuclear envelope.
\end{abstract}

Intercellular signaling in the nervous system is mediated at the synapse, a highly specialized structure that has evolved to transform electrical information carried by the action potential to a secreted chemical message (e.g., Augustine et al., 1987). Yet despite its central role in the nervous system, little is known about many of the molecules that underlie presynaptic function (Reichardt and Kelly, 1983). A number of molecules that are localized to the presynaptic nerve terminal have been identified, including synapsin I (Huttner et al., 1983; McCaffery and DeGennaro, 1986; Baines, 1987), tor 70 (Carlson and Kelly, 1983), synaptophysin (Matthew et al., 1981; Buckley and Kelly, 1985; Wiedenmann and Francke, 1985), calelectrin (Geisow et al., 1986), and mediatophore (Israel et al., 1986). However, the precise roles of these molecules in synaptic transmission are still under investigation.

Of particular importance in communication between neurons is the metabolism of neurotransmitters which must be synthesized, packaged into vesicles, transported, and released at the correct time and place. Modulation of these processes generates changes in the efficacy of synaptic connections and underlies simple forms of learning. Both short-term changes dependent on second-messenger-mediated covalent modification of preex-

\footnotetext{
Received July 27, 1987; revised Nov. 27, 1987; accepted Dec. 10, 1987.

We would like to thank David Cowan, Cindy Murphy-Erdoch, and William Trimble for assistance with several of the experiments reported in this manuscript and Reg Kelly for the synaptic vesicle antibody. We also thank Corey Goodman and William Trimble for critically reading the manuscript. L.M. is supported by the CNRS and by a grant from EMBO. This work is supported by grants to R.H.S. from the NIMH. R.H.S. is a PEW Scholar and the recipient of a Presidential Young Investigator Award

Correspondence should be addressed to Dr. Richard H. Scheller at the above address.

Copyright (C) 1988 Society for Neuroscience $0270-6474 / 88 / 082804-12 \$ 02.00 / 0$
}

isting molecules and long-tcrm changes in gene expression have been proposed to account for the modulation of synaptic strength (Berridge, 1986; Goelet et al., 1986; Schwartz and Greenberg, 1987), yet the means whereby synaptic and nuclear events are coordinated remains a mystery. By defining neuron-specific gene products localized to the synapse we hope to further our understanding of these and other neuronal functions.

A difficulty in defining the molecular architecture of the synapse lies in the heterogenous nature of this complex structure in the CNS. The electromotor system of the marine elasmobranchs provides a partial solution to this problem. The electromotor nucleus is a large homogenous cluster of cholinergic motor neurons innervating the electroplate cells of the electric organ (Luft, 1958). The electric organ contains an extremely high density of synapses, which closely resemble the mammalian neuromuscular junction. This system has been exploited in studics of scveral postsynaptic molcculcs, including the ACh receptor (Noda et al., 1982) and AChE (Schumacher et al., 1986).

The electric organ is an important source of materials for studies of the presynaptic nerve terminal as well. Cholinergic synaptic vesicles from electric organ have been purified and characterized in some detail. The vesicles from Narcine brasiliensis are $80 \mathrm{~nm}$ in diameter and contain approximately 47,000 molecules of ACh, as well as 17,000 molecules of ATP and GTP. The purified synaptic vesicles have a lipid-to-protein ratio of $5: 1$ by weight, and 20 size classes of proteins are observed on SDS/polyacrylamide gels (Carlson et al., 1978; Tashiro and Stadler, 1978; Wagner et al., 1978; Ohsawa et al., 1979). While some of these proteins appear specific to the purified vesicles, others appear in the nonsynaptic vesicle material as well.

Carlson and Kelly (1980) generated a rabbit antisera by immunizing with purified cholinergic synaptic vesicles from electric rays. When this antisera was preabsorbed with electric organ membranes free of synaptic vesicles, about half of its vesicle binding activity was retained. Therefore, the vesicle preparations contain both antigens which are shared with other membranes and those specific to synaptic vesicles. Additional studies demonstrated that this antibody stains a subclass of mammalian nerve terminals (Hooper et al., 1980).

To further characterize synaptic proteins we used this antisera against cholinergic vesicles to screen an expression vector cDNA library constructed with mRNA isolated from the electromotor nucleus. In this report we characterize one of the genes isolated from this screen. In Torpedo and rat the gene encodes a protein, synuclein, comprised largely of 11 amino acid repeat units. The gene is expressed only in neurons, and immunoreactivity is localized to a region of the nucleus as well as the presynaptic nerve terminal. On the basis of the subcellular localization of 
immunoreactive material, we propose that this protein, or family of proteins, may be involved in coordinating nuclear and synaptic events.

\section{Materials and Methods}

cDNA cloning. Clones were obtained by expression screening of a cDNA library made in $\lambda \mathrm{gt} 11$ from poly $\mathrm{A}^{+}$RNA isolated from the electric lobe of Torpedo californica. The antibody used was raised against purified synaptic vesicles by Carlson and Kelly (1980). Library preparation and isolation of clones were performed essentially as published (Huynh et al., 1985).

Antisera preparation. Fusion protein was made from both clone $17 \mathrm{~B}$ and $\lambda \mathrm{gt}-11$ phage without insert. Crude lysate was run on polyacrylamide-SDS gel, and the fusion protein was excised from the gel, washed in distilled water and stored at $-20^{\circ} \mathrm{C}$. Fisher strain rats were injected with $40 \mu \mathrm{g}$ protein in the gel slice, intraperitoneally 5 times at $10 \mathrm{~d}$ intcrvals. Blood was collected by cardiac puncture, spun at $10,000 \times g$ for $30 \mathrm{~min}$ at $4^{\circ} \mathrm{C}$, and stored frozen at $-80^{\circ} \mathrm{C}$ until use.

Microscopy. Torpedo tissues were fixed and embedded in Epon or in Lowicryl $\mathrm{K} 4 \mathrm{M}$ and immunostained according to published procedures (Kreiner et al., 1986), except that Torpedo physiological saline buffer ( $280 \mathrm{~mm} \mathrm{NaCl}, 3 \mathrm{~mm} \mathrm{KCl}, 1.8 \mathrm{~mm} \mathrm{MgCl}$, $1.2 \mathrm{~mm} \mathrm{NaPi}$, pH 6.8: TPS) was used instead of PBS. Primary antibody was diluted 1:100 for immunofluorescence, and 1:10 for EM immunochemistry.

Protein blotting. Proteins were extracted in the presence of $10 \mathrm{~mm}$ EGTA, according to Wagner et al. (1978). Blotting on nitrocellulose filters was carried out essentially as Burnette (1981), and revealed by HRP-DAB method.

$R N A$ analysis. RNA was extracted in the presence of guanidiniumHCL, poly $\AA^{+}$RNA was selected on oligo dT columns and separated on formaldehyde agarose gel using standard procedures (Maniatis et al., 1982).

DNA sequencing. After subcloning into Bluescript vectors, chain-termination methods of sequencing were used with Klenow enzyme and later with Sequenase enzyme according to the manufacturers recommendations.

\section{Results}

\section{The synuclein protein}

About $400,000 \lambda \mathrm{gt}-11$ clones were screened with the antisera generated against synaptic vesicles, resulting in about 150 positive plaques with signals of varying intensity above background. Forty-nine clones with a variety of signal intensities were purified. To determine the number of independent sequences present in this set of clones, individual cDNA inserts were isolated and hybridized back to the original set of clones. The 49 clones fell into 17 classes: two clones were represented 10 times, one clone 6 times, 3 clones 3 times, 3 clones 2 times, and 8 clones were each present once. The inserts range in size from 500 nucleotides to about 3 kilobases $(\mathrm{kb})$. Since almost half of the clones were isolated only one time, further screening would be required to purify all of the genes in the library that synthesize fusion proteins recognized by the synaptic vesicle antibody.

The original Torpedo cDNA clone (clone 17B, present 6 times in the original screen) was used to screen a rat brain cDNA library, resulting in several clones which hybridized at an intermediate stringency of $60^{\circ} \mathrm{C}$. The nucleotide and inferred amino acid sequences of the rat and Torpedo cDNAs are shown in Figure 1. The rat synuclein cDNA clone contains a long 5 ' untranslated region of almost 1500 nucleotides. This region contains numerous stop codons in all reading frames; the longest potential protein that could be encoded by this portion of the cDNA is only 84 amino acids. In contrast, the Torpedo cDNA clone contains $5^{\prime}$ untranslated region that begins at a natural EcoRI restriction enzyme site 27 nucleotides from the initiator methionine residue. Thus, the Torpedo $\beta$-galactosidase ( $\beta$-gal) fusion protein consists of 9 amino acids encoded by the un- translated region of the cDNA followed by the 143 amino acids of synuclein (Figs. 1 and 2). The 3' untranslated regions of the rat and Torpedo clones are 268 and 441 nucleotides, respectively. No significant nucleotide sequence homology is observed between these untranslated regions. Open reading frames 143 and 140 amino acids are found in the Torpedo and rat clones. The 2 cDNA clones are about $85 \%$ homologous throughout much of the open reading frame, confirming this portion of the cDNAs as the coding region.

The Torpedo and rat synuclein proteins have calculated molecular weights of 14,811 and 14,496 Da, respectively, and are schematically illustrated in Figure 2. Several copies of an 11 amino acid repeat are apparent in the synuclein protein as depicted in Figures $1-3$. The first repeat begins 6 amino acids after the initiator methionine and is followed by 7 tandem copies in rat $(\mathrm{R} 1-7)$ and 8 tandem copies in the Torpedo (T1, T2a, T2b, T3-7) protein. Homologous repeat units between species are more highly conserved than units within a particular synuclein protein. For example, T2 and R2 are more homologous than $\mathrm{T} 1$ and $\mathrm{T} 2$. This organization suggests the repeated motif arose prior to the divergence of rat and Torpedo. In contrast, repeat units $\mathrm{T} 2 \mathrm{a}$ and $\mathrm{T} 2 \mathrm{~b}$ are identical in both protein and DNA sequence, and only a single copy of this repeat is found in the rat gene, suggesting the second copy of this unit arose via a relatively recent duplication since the divergence of Torpedo and rat.

The 11 amino acid repeat sequences are aligned in Figure 3, revealing a central conserved core of 6 amino acid residues, LysThr-Lys-Glu-Gly-Val. These amino acids are present in at least 10 of the 14 possible positions, whereas the first 3 and the last 2 residues of the repeat unit are more highly variable. Due largely to the presence of 3 charged residues in the conserved core, most of the repeated region of synuclein is hydrophilic and basic. Unit 6 is the least conserved of the repeats, where all of the charged residues are replaced by hydrophobic or polar amino acids. As a result, the carboxy terminal end of repeat 5 , repeat 6 , and the amino terminal end of repeat 7 form an 18 amino acid stretch lacking charged residues. The presence of a number of valine and alanine residues in this portion of the protein gives rise to a substantial hydrophobic region. The homology between species over the repeated region of the gene is about $80 \%$, however, the homology drops quite dramatically as one moves further toward the carboxy terminus of the protein. While the precise sequence of the carboxy terminal end of the protein is not well conserved between species, the hydrophilic and acidic nature of this region is conserved.

Only one homology to other characterized sequences has been found by searching protein data banks. The 6 amino acids of the conserved core are found in the carboxy terminal portion of the rho gene family. The rho genes share $35 \%$ homology with the ras family of $\mathrm{G}$ proteins (Madaule and Axel, 1985), which may be involved in transducing information from cell surface receptors (Gilman, 1984; Michell and Kirk, 1986). The homology occurs in a portion of the ras and rho genes which are relatively conserved and have been proposed to form part of the GDP binding pocket (McCormick et al., 1985).

\section{Expression of the symuclein gene}

RNA blotting was used to determine the tissue specificity of synuclein expression. Poly $\mathrm{A}^{+}$RNA was isolated from several Torpedo tissues and probed with a Torpedo actin gene to assess the state of the RNA in the various preparations (Fig. $4 A$ ). Each of the 6 RNA samples hybridized to an actin transcript of ap- 
2806 Maroteaux et al. - Nuclear and Presynaptic Terminal Protein

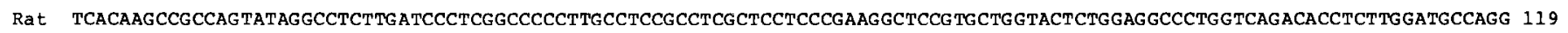

Rat ACATCAGgGGTGACATGACGTTCACTCCAGCCTTGCTGGCCACCCACCGGGCCTTCACCTCCACTCCTGCCCTGTTTGGGCTTGTGGCTGACAGGCTGGAAGCCTCGAATCGCATCC 23 8

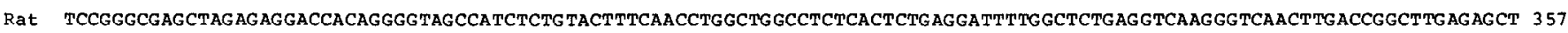

Rat TCTTGCTTCGGACAGGGAGCTGTTTCTCAGTCTGATCCCCTCTCAGTGTTTGGGAGGCCTATGGGGTATAGAGACCGGCCAGGACATTCTCATCTCTGCCCGTCTGTCCGAGCTACCG 476

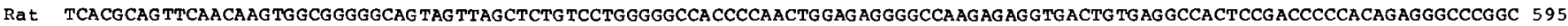

Rat TCCTGGAGAAGTGGATCCGGTCTTAGGCAATGTGTTCCCTTGAGAAGGTCAGTCCTTCCCCCAACCTTGGCTCTGACAAGGTTCTGTCATCTGTCTATCTTCTTTCTGTCTGTCTG 714

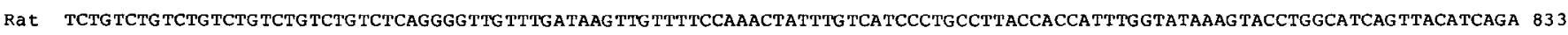

Rat CAATTCTAATGGAATGTTGCTTCTCCTGAAGGCTTTTCTTCAGCTTCATCATTGGAAGgAAAGAAATAATAACAGAGACATAACTCTACAAACTACACTAACCTAGTTCCCTGCTCCA 952

Rat AaCTCCGgAAAACTGTGTCTTAACTGGgACATGgAAATACAGTAATCAACCCCCATCTTCAGTTCTGCATGACTCCATTTCAATTACCTGTAGACAACCATGATCCAGAAATATTTATA 1071

Rat TTTGTCTTAACTCTTTTGAGAGAGTCCACAATTACATAGCTTTTATTACACTATATTCTTATAACTTGCCTACTTTATTATTGTCAATCTCTTTCTG TGCCTAATTACAAATTAAAC 1190

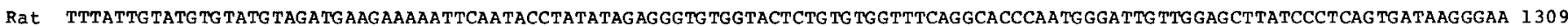

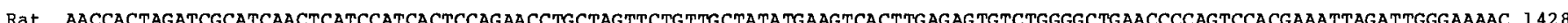

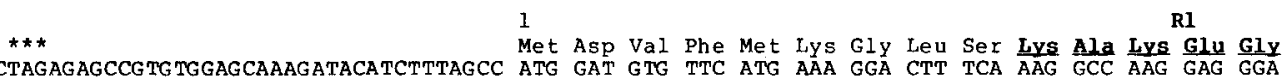

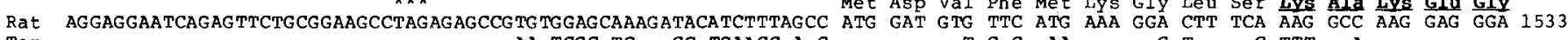

Tor$$
\text { R2 }
$$

Val Val Ala Ala Ala Glu Lys Thr Lys Gln Gly Val Ala Glu $/ / / / / / / / / / / / / / / / / / / / / / / / / / / /$ Ala Ala Gly Lys Thr

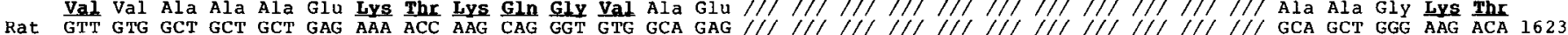

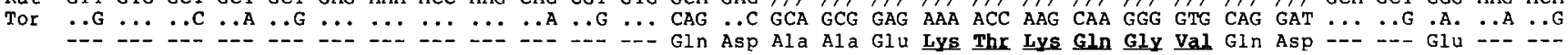
45 R3

45 R3
Lys Glu Gly Val Leu Tyr Val Gly ser Lys Thr Lys Glu Gly val val His Gly Val Thr Thr Val Ala Glu tys Thr Lys Glu Gln Val
AAA GAG GGC GTC CTC TAT GTA GGT TCC AAA ACT AAG GAG GGA GTC GTT CAT GGA GTG ACA ACA GTG GCT GAG AAG ACC AAA GAG CAA GTG 1713

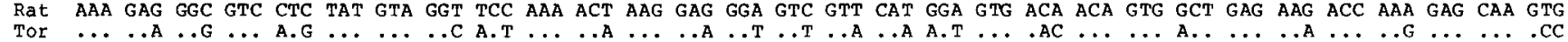

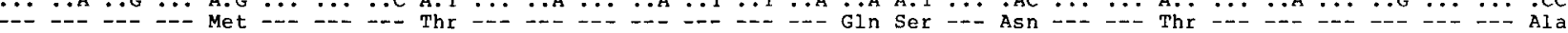

$7.5 \quad$ R6 R7

Thr Asn Val Gly Gly Ala Val Val Thr Gly Val Thr Ala Val Ala Gln Lys Thr Val Gly Gly Ala Gly Asn Ile Ala Ala Ala Thr Gly

Rat ACA AAT GTT GGA GGG GCA GTG GTG ACT GGT GTG ACA GCA GTC GCT CAG AAG ACA GTG GAG GGA GCT GGG AAC ATT GCT GCT GCC ACT GGT 1803

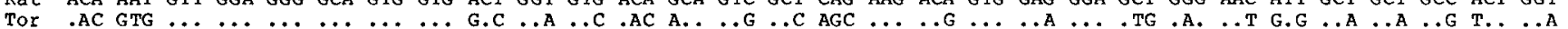

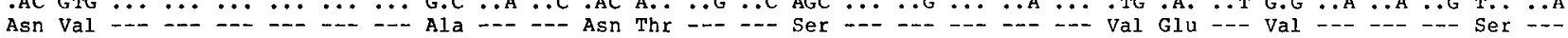
105

Phe Val Lys Lys Asp Gln Met Gly Lys Gly Glu Glu Gly Tyr Pro gln Glu Gly Ile Leu Glu Asp Met Pro Val Asp pro Ser Ser Glu Rat TTT GTC AAG AAG GAC CAG ATG GGC AAG GGT GAA GAA GGG TAC CCA CAA GAG GGA ATC CTG GAA GAC ATG CCT GTG GAC CCT AGC AGT GAG 1893

Tor G.G ..G ..A TT. ..T G.A CAT ..T . GA $/ / /$... ATC CCT GCT GA. ..G .TG .CT GAG GGA A.. C.G .CC A.. CA. ..A ..C CT. GTG ...A

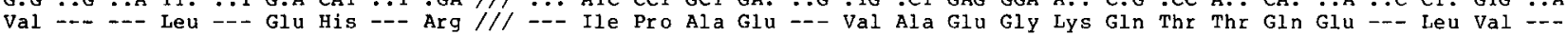

135

Ala Tyr Glu Met Pro Ser Glu Glu Gly Tyr Gln Asp Tyr Glu Pro Glu Ala ***

Rat GCT TAT GAA ATG CCT TCA GAG GAA GGC TAC CAA GAC TAT GAG CCT GAA GCC TAA GAATGTCGTTGCACCTACTGTCCTAAGATCTGCCCAGGTGTTCTTCC 1994

Tor ... ACA ... GCA A.. GA. ... ACT ... A A. A TG. . CA CC. ACT GA. TTC C. G A.g ATTCTGG. . GCACTTGC.CTCT.CCCTTAACCAT..CACCAGTG. TG

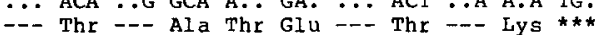

Rat ATGGCG TACAAGTGCTCAGTTCAACGTGCCCAGTCATGACCTTTTCTCAAAGCTG TACAGTG TATTTCAAAGCTTCCATCAGCAGTGATCGGAGTCCTGTACCTGCCCTCAGCATC 2113

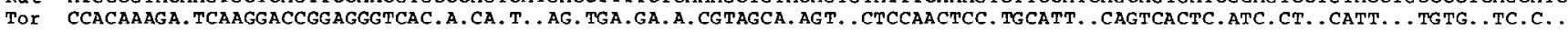

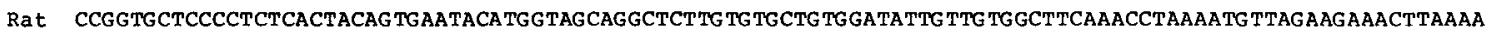

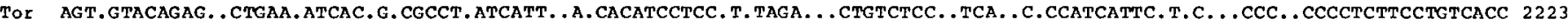

Tor TGATCACACCAATGTTCAACTTG TGATgGGCAATGCA TG TTCTTCACTGGCTTTG TG TG TG TGGCATTAACTG TTG TGAGCACTTGCAGATTTATAATAAATGCAAAATAAAA 2351

Tor

Figure 1. Nucleotide and predicted amino acid sequence of rat and Torpedo synuclein. The nucleotide sequences of rat brain (top) and Torpedo electric lobe (bottom) cDNA clones are presented along with the predicted amino acid sequences. The complete rat sequence is shown, and the differences between the species are presented below in the lines labeled Tor. Nucleotides are numbered to the right and the amino acids are numbered above. Two insertions and/or deletions were introduced to maximize the homology $(/ / /)$. Termination codons flanking the coding regions are illustrated in the $5^{\prime}$ and $3^{\prime}$ ends of the cDNAs $\left({ }^{* * *}\right)$. The AXUAAA sequence is underlined in the 3 ' end of the Torpedo clone. The amino acids of the conserved core region of an 11 amino acid repeat is bold and underlined. The repeats are numbered R1-7, corresponding to the 7 repeats of the rat sequences. The Torpedo protein contains a unit that is not present in rat, labeled $\mathrm{T} 2 \mathrm{~b}$.

proximately $2.1 \mathrm{~kb}$. Differences in the intensity of hybridization may reflect different levels of actin transcripts, the relative purity of the poly $\mathrm{A}^{+} \mathrm{RNA}$ or some combination of these and other variables. The actin probe was washed off the filter and reprobed with the labeled synuclein cDNA insert. Figure $4 B$ demonstrates synuclein is expressed in the electric lobe, the brain, and the spinal cord (lanes 1,2 , and 5) but not in muscle, liver, or electric organ (lanes 2, 4, and 6). The transcript is approximately 1.4 $\mathrm{kb}$, and no hybridization is seen in the non-neural tissues even with much longer exposures. Relative to the actin transcript, the synuclein message is most abundant in the electric lobe followed by spinal cord and brain, suggesting the gene is not expressed at equivalent levels in all CNS tissues. Similar experiments in rat demonstrate the gene is expressed in brain but not spleen, liver, heart, or kidney (data not shown). Therefore, the data thus far demonstrate nervous system specific expression of synuclein.

The distribution of synuclein immunoreactivity was studied using protein blotting techniques (Fig. 5). Protein was extracted, separated by PAGE, blotted onto nitrocellulose, and probed with an antiserum raised against the Torpedo synuclein $/ \beta$-gal fusion protein or native $\beta$-gal. Inspection $8 \%$ polyacrylamideSDS gel blots did not reveal any differences between the fusion protein antibody and the control. In contrast, blots of $15 \%$ poly- 


\section{TORPEDO}

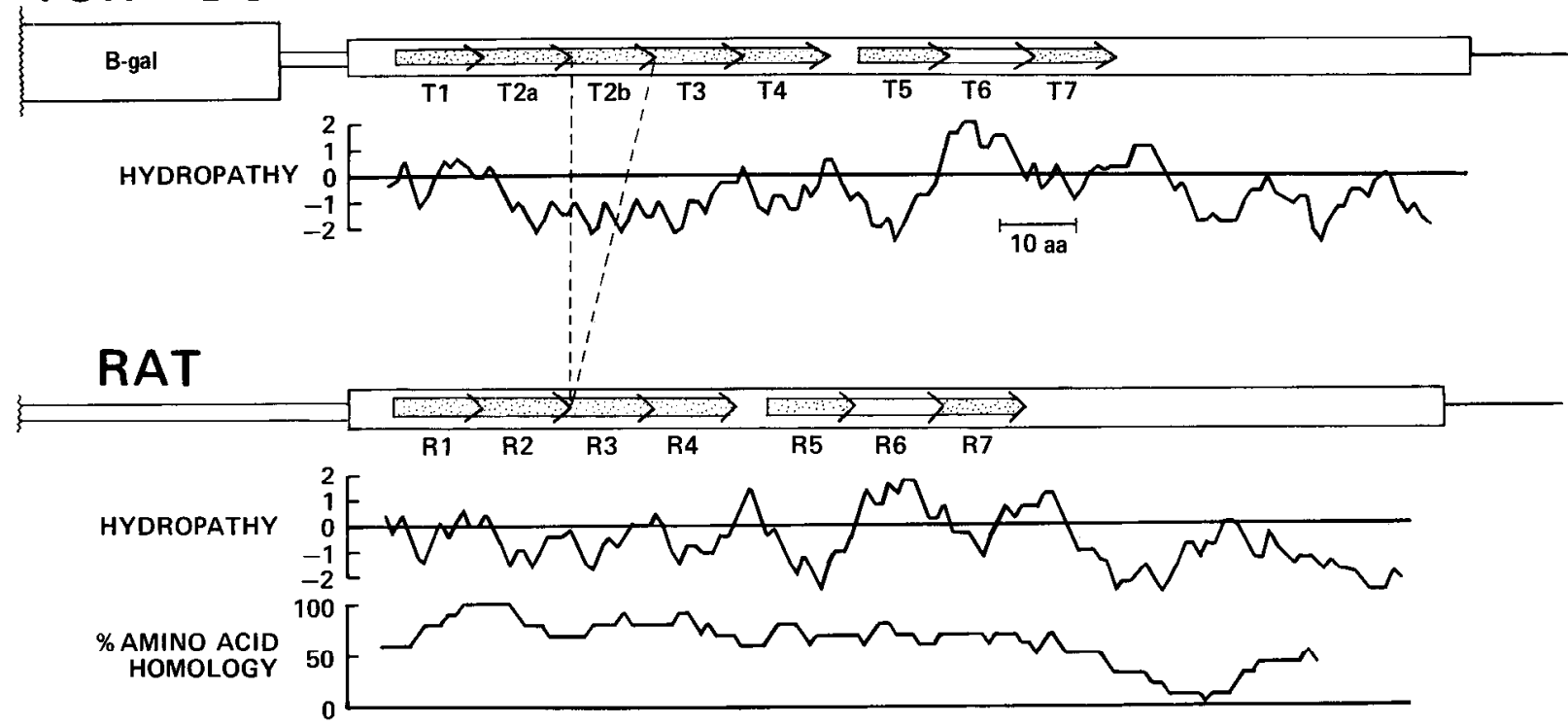

Figure 2. Schematic representation of the rat and Torpedo synuclein proteins. The $\beta$-gal/synuclein fusion protein contains 9 amino acids that are synthesized from the $5^{\prime}$ untranslated region of the mRNA followed by the complete Torpedo protein. The 11 amino acid repeating unit is represented by arrows and the individual repeats are labeled $\mathrm{T} 1, \mathrm{~T} 2 \mathrm{a}, \mathrm{T} 2 \mathrm{~b}$ and $\mathrm{T} 3-\mathrm{T} 7$. T2b is not present in the rat protein. The hydropathy plot shows that the protein is largely hydrophilic with the exception of the region centered around repeat T6. A schematic of the rat protein is presented along with the corresonding hydropathy plot. The percentage amino acid homology between rat and Torpedo synuclein is plotted using 10 amino acid windows along the length of the protein. The portion of the protein containing the repeat is highly conserved. While the $3^{\prime}$ ends are both acidic, the precise sequence is not well conserved.

acrylamide gels containing urea to resolve the low-molecularweight proteins (Kaldany et al., 1985) revealed a set of specific bands in the molecular-weight range between 17.5 and $20 \mathrm{kDa}$. The electric lobe, spinal cord, and brain exhibit an intensely staining band at $17.5 \mathrm{kDa}$ and a series of fainter bands at 18.5 and $20 \mathrm{kDa}$, as well as less abundant higher-molecular-weight species. No specific staining was observed in heart (data not shown), muscle, or liver. Three bands of approximately equal intensity were observed in the electric organ. Since no synuclein RNA is observed in the electric organ, the proteins must be synthesized in the neuronal cell bodies and axonally transported to the nerve terminals. In summary, synuclein is a protein or series of proteins expressed in the nervous system but not in a variety of other tissues in Torpedo and rat. In addition, the proteins are transported to nerve terminals and are found in at least 3 different forms.

\section{Synuclein in the neuronal cell soma}

The distribution of synuclein-immunoreactive material was investigated using both light and EM level immunohistochemistry. Electric lobe tissue was fixed in paraformaldehyde followed by embedment in Lowicryl. One micron sections were stained with synuclein fusion protein antibody and fluoresceinconjugated wheat germ lectin, which recognizes sugar residues, added to proteins in the Golgi (Vanen et al., 1980). A goat anti-rat antibody coupled to rhodamine was used to visualize the synuclein immunorcactivity (Fig. 6). These experiments revealed intense staining, not observed in control experiments with the $\beta$-gal antibody, which appeared to be along a restricted segment of the inner nuclear membrane. This staining was observed in over 50 independent neurons, but only in about $15 \%$ of the sections containing neuronal nuclei. The fraction of neurons staining for synuclein can, at least in part, be attributed to the fact that only part of the nucleus contains immunoreactive material.

To better resolve the immunoreactive material, EM histochemical studies using HRP to visualize the antigen were per-

1 11

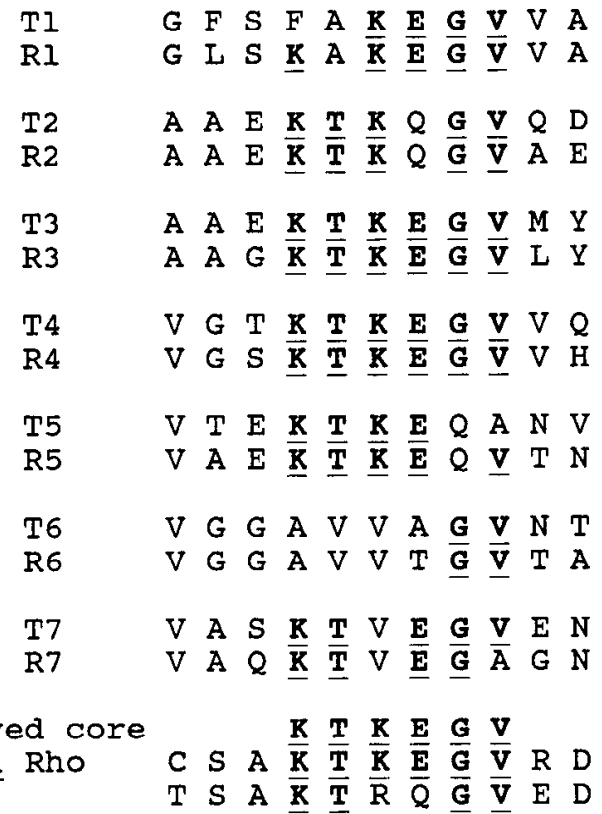

Figure 3. Alignment of the rat and Torpedo 11 Amino Acid Repeat Sequence. The 7 rat (R1-7) and Torpedo (T1-7) repeat sequences are aligned, revealing a highly conserved central core of 6 amino acids, which are bold and underlined. Residues of the conserved core are homologous at a minimum of 10 of 14 positions, while other positions are less well conserved. A homology to the Aplysia rho and human ras proteins is presented. 


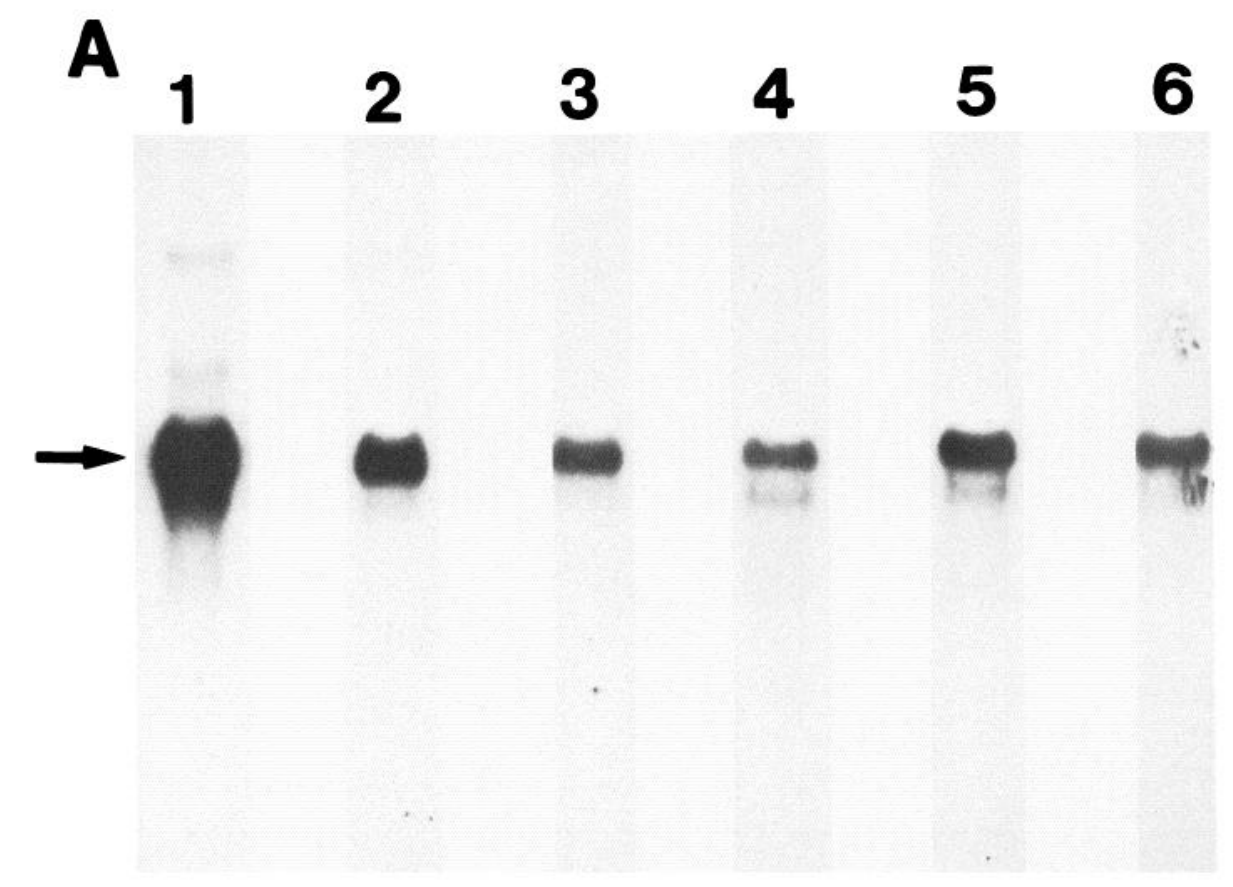

Figure 4. Synuclein is specifically expressed in neural tissues-RNA blot analysis. $A$, Poly $\mathrm{A}^{+}$RNA was purified from electric lobe (lane 1), brain (lane 2), muscle (lane 3), liver (lane 4), spinal cord (lane 5), and electric organ (lane 6 ). RNA, $6 \mu \mathrm{g}$, was fractionated on agarose gels, transferred to nitrocellulose and probed with a radiolabeled Torpedo actin gene. The arrow indicates a transcript of approximately $2.1 \mathrm{~kb} . B$, The blot in $A$ was washed of bound actin probe and rehybridized to a labeled synuclein cDNA clone. The large arrow indicates a $1.4 \mathrm{~kb}$ transcript found in the electric lobe, brain, and spinal cord; the smaller arrow indicates the position of the actin transcript.

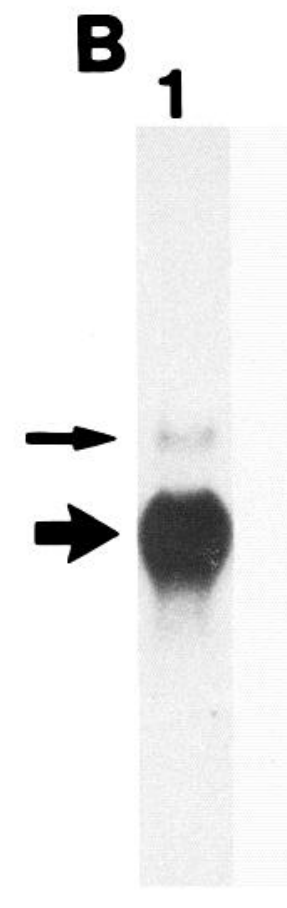

\section{2}
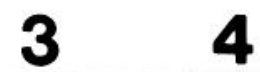

5

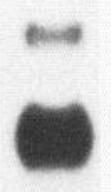

formed. In these experiments, the precise localization of the antigen was more apparent. A portion of the nuclear envelope is most intensely stained and the immunoreactivity diminishes toward the center of the nucleus. In this section, the staining is along $90 \mu \mathrm{m}$ of nuclear membrane and the gradient is approximately $10 \mu \mathrm{m}$ deep, although this varied somewhat from cell to cell (Fig. 7).

Occasional punctate cytoplasmic staining was observed with a control antibody generated against $\beta$-gal, and therefore it is not specific to synuclein. Furthermore, it is possible that an antigen unrelated to the gene we have cloned is being recognized in this experiment. The agreement between the predicted mo- lecular weight of the protein from the cDNA sequence and the protein blots described earlier suggests the same antigen or antigens are being recognized in the terminals and the cell body. In further summary, these experiments suggest that in the cell bodies of the electromotor neurons synuclein immunoreactivity is localized to a restricted region of the nucleus initiating along the nuclear envelope.

\section{Synuclein in the presynaptic nerve terminal}

Light level immunofluorescence of the electric organ is shown in Figure 8. The organization of the electroplate cells cut in cross section is evident as parallel membranes consisting of alternately 

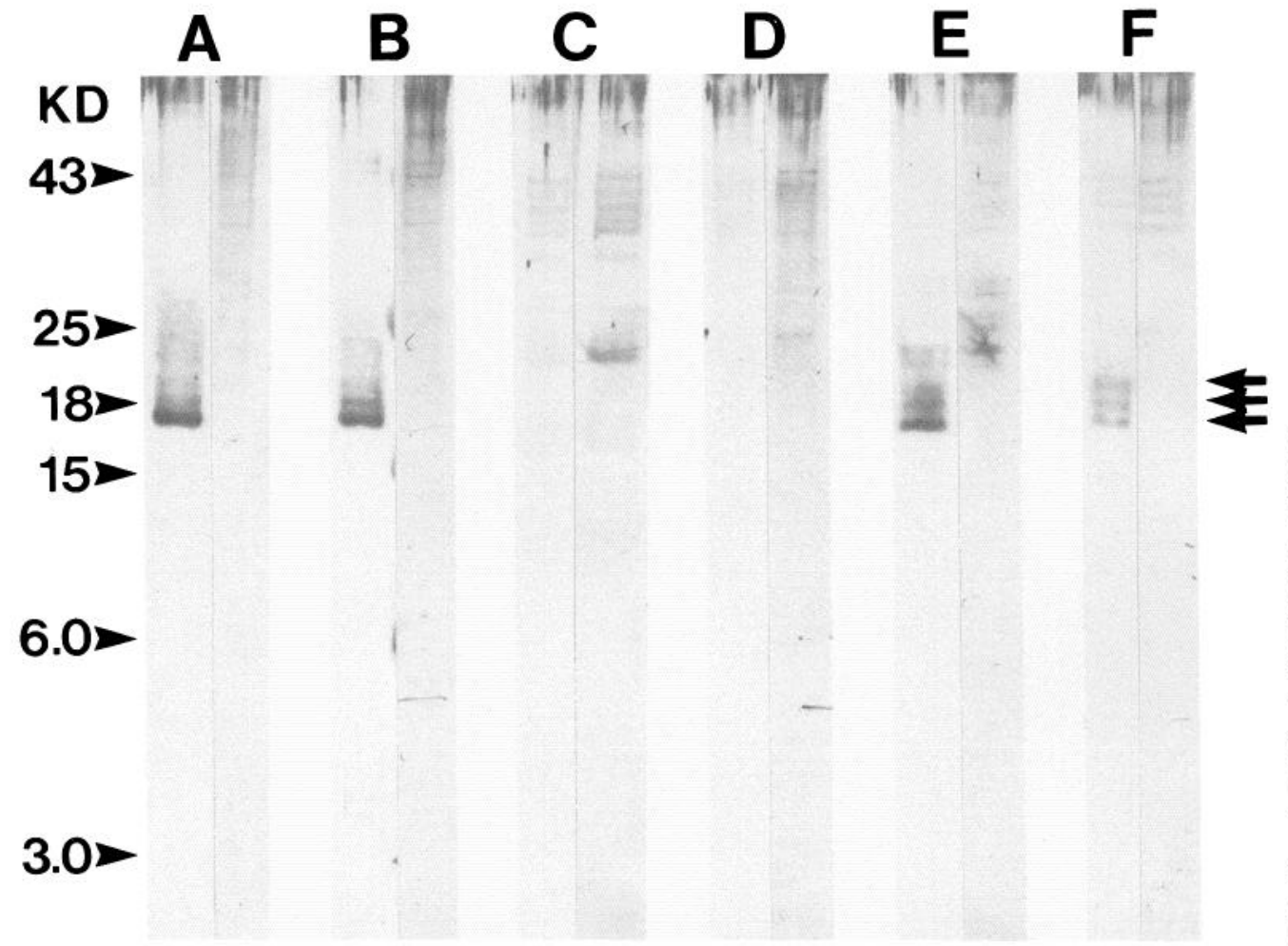

Figure 5. Synuclein is transported to the electric organ-protein blot analysis. Proteins were extracted as described in Materials and Methods and separated on $15 \%$ polyacrylamide gels containing $0.1 \%$ SDS and $8 \mathrm{~m}$ urea. Each sample was probed with both anti $\beta$-gal (right) and anti $\beta$-gal/synuclein fusion protein (left). Electromotor nucleus (lane $A$ ), brain (lane $B$ ), and spinal cord (lane $E)$ all contain a prominent band at 17.5 $\mathrm{kDa}$ (bottom arrow at right). Electric organ (lane $F$ ) contains 3 immunoreactive species of molecular weights 17.5 , 18.5 , and $20 \mathrm{kDa}$. Muscle (lane $C$ ) and liver (lane $D$ ) do not contain any immunoreactive bands not seen in the $\beta$-gal control.

innervated and noninnervated faces (Luft, 1958). In Figure $8 \mathrm{~A}$ the use of fluorescein-conjugated lectin displays the plasma membrane probably due to the high concentration of membrane glycoproteins. The synuclein fusion protein antibody stains in a punctate pattern along every other membrane. The punctate staining, consistent with a localization to presynaptic nerve terminals, is more evident in the section shown in Figure $8 B$. As in the Western blotting and cell soma immunohistochemistry, the anti $\beta$-gal antibody was used as a control in this series of experiments. No staining at either the light or EM level was observed with the control antibody. Staining of axons was slightly above background, suggesting axonally transported immunoreactive material is concentrated in the synapse.

The localization of immunoreactive material in the electric organ was investigated at higher resolution using EM histochemical experiments with colloidal gold to visualize the antigen. Figure $9 A$ shows a section through the innervated face of the electric organ revealing the salient features of the synapse, including the presynaptic terminal containing vesicles and mitochondria. The junctional fold is apparent as an indentation in the electric organ opposite the presynaptic terminal. To preserve antigenicity, electric organ tissue was embedded in Lowicryl followed by reaction with the synuclein fusion protein antibody and protein A bound to $10 \mathrm{~nm}$ colloidal gold (Fig. $9 B$ ). The experiment clearly shows a large number of gold particles in the presynaptic terminal and relatively few in the electroplaque cells. Since the sections were dehydrated in ethanol, the membranes are not well preserved, making it difficult to determine the precise localization of the antigen with respect to the vesicles. The circular profiles do, however, represent the remnants of vesicles and are indeed associated with the majority of gold particles in the terminal. The resolution in this experiment is insufficient to determine if the antigen is localized to the inside or the outside of the vesicles.

\section{Discussion}

Synuclein: repeated protein structure and cellular localization The synuclein gene is specifically expressed in the CNS of Torpedo and rat, and synuclein/ $\beta$-gal immunoreactivity is localized to a restricted area of the nucleus and to the presynaptic nerve terminal. The predicted protein consists of an 11 amino acid sequence that is repeated 7 or 8 times, followed by a $40-50$ residue acidic tail. The repeats contain a conserved core which is also found in the rho gene family. Since many different types of proteins, ranging from neuropeptide precursors (Taussig and Scheller, 1986) to intermediate filament proteins (Myers et al., 1987), and extracellular matrix molecules contain internally repeated units, this aspect of the structure does not define a function. This highly repeated structure does, however, suggest that synuclein probably does not fold into a molecule capable of catalytic function.

The protein blotting experiments demonstrate the presence of at least 3 forms of synuclein, each of which differs in apparent molecular weight by approximately $1000 \mathrm{Da}$. The lowest molecular weight species is most abundant in the electric lobe, the brain, and the spinal cord and may represent the unmodified protein. The higher-molecular-weight forms may represent progressively increasing modifications of individual repeat units. The increased fraction of the higher-molecular-weight forms in the synapse may be the result of processes that occur during axonal transport or actually in the synapse itself.

In the neuronal cell soma, synuclein fusion protein immunoreactivity is localized along a patch of nuclear membrane. The concentration of immunoreactive material dissipates towards the inside of the nucleus. Immunohistochemical investigations of the localization of protein kinase $\mathrm{C}$, which may be involved in neurotransmitter metabolism (Kaczmarek, 1987), demonstrate nerve terminal staining and a similar distribution 


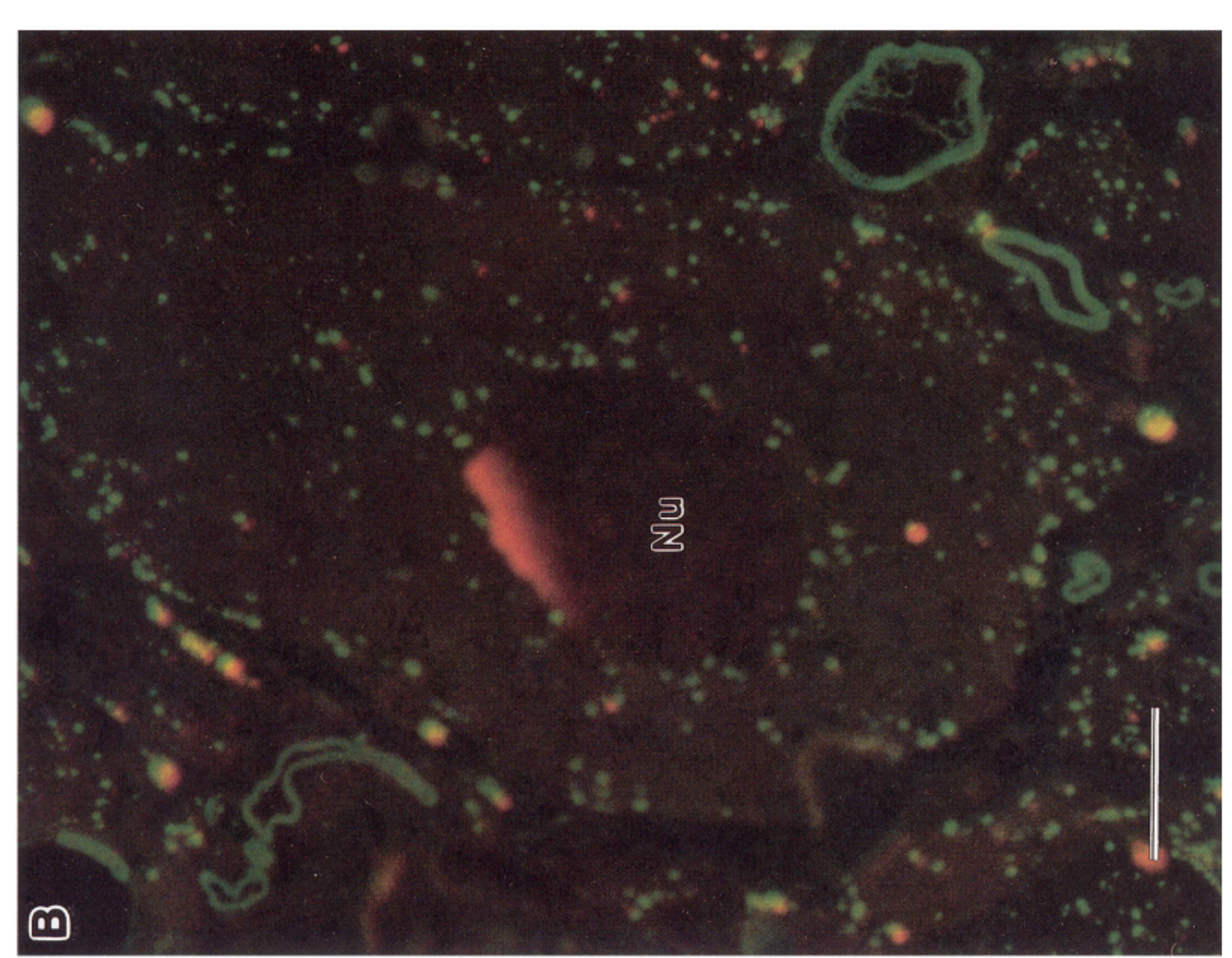

客:

융충

运

.

论

记

.

边

독

है

उ。

:

릉.

.

帘

两学

ob

青 8

픔 윰

․ㅡㅁ 동

淽里

ते.ंె口

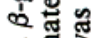

可

․․․

$F$.

ช்

ํํㄹ

䝿品

․․․

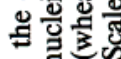

घ

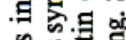

号跑.

봉

I

농

on

군

政

을

8 툰

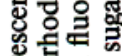

$0<$

压娄

$\infty$ 응

항

政

$\checkmark$.

을

둴 동

운 क्ष

政

당 웡으

용

可可

o

.

둥

क्ष

品额

हत

कि焉

6 .

․․․․․

(1)

㕸 

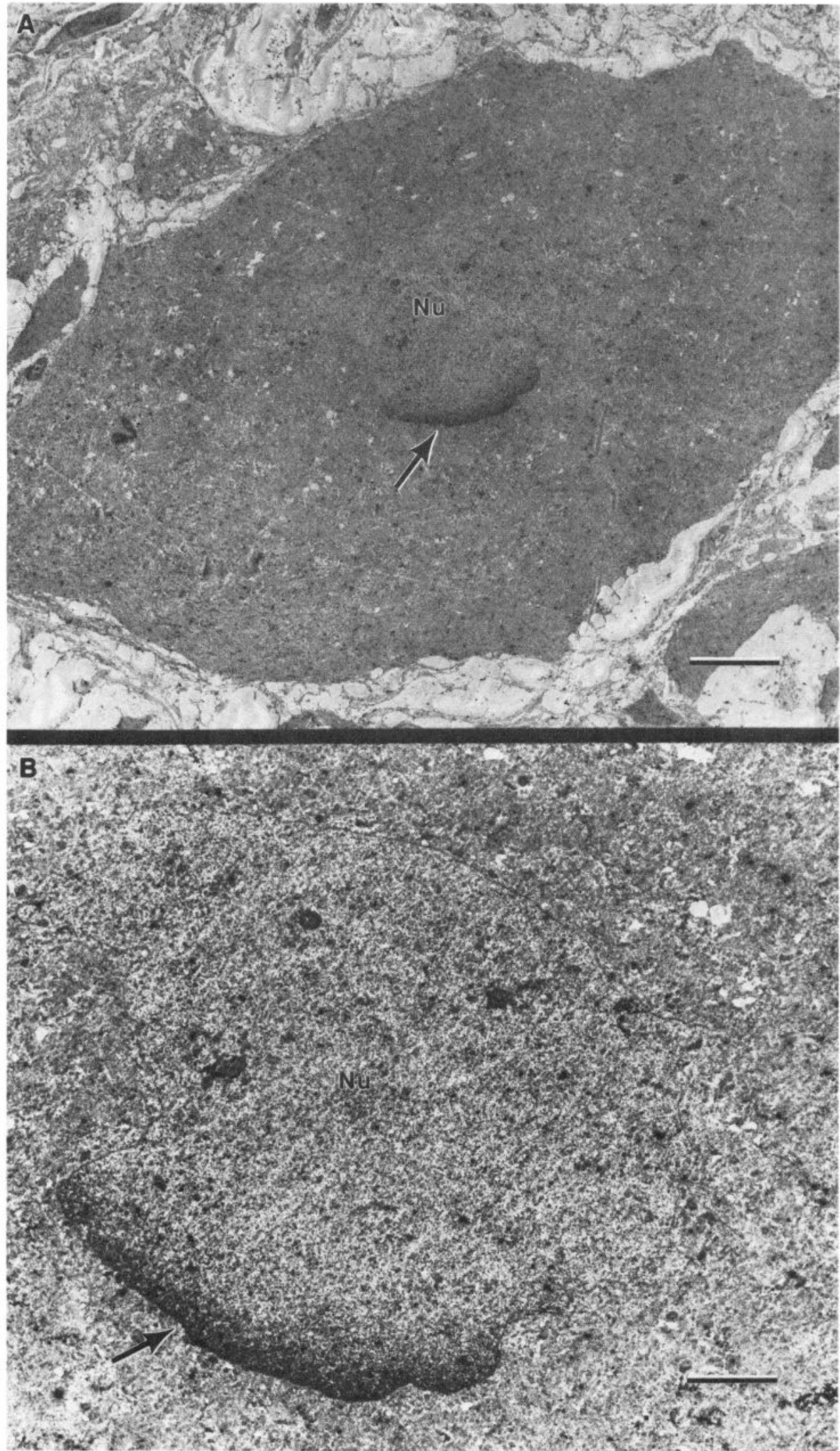

Figure 7. Electron microscopic resolution of synuclein in neuronal nucleiimmunoperoxidase staining of electric lobe neurons. $A$, Section, $70 \mathrm{~nm}$, stained with antifusion protein antibody. The only significant staining is localized to a portion of the nucleus beginning at the nuclear membrane (arrow). Scale bar, $13.5 \mu \mathrm{m}$. $B$, Higher magnification of the section shown in $A$. The staining is localized in a gradient about $10 \mu \mathrm{m}$ wide. Scale bar, $4.6 \mu \mathrm{m}$. along the nuclear membrane (Wood et al., 1986; Cambier et al., 1987). However, in contrast to synuclein, in some experiments the kinase is found around the entire nucleus (Wood et al., 1986). Thus, synuclein defines a distinct polarization of the neuronal nucleus. We do not yet know if this polarization is specifically oriented with respect to other landmarks in the neuron such as the axon or dendrites.

Two types of molecular interactions may account, at least in 
政

욜. 근

琼

自究

둥

응

क्

目

品婇

击

政

3 o

을

\&졸명

要击

एक

负

政

엠

도임

동 홍.

희

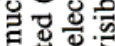

今

品

b

7 요

สㅇํㅇ

40

近证



2.․․․

×. 专.

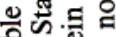

\% 8

○幽

ते

ن

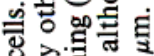

o

和数

挣.

매을

边

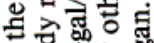

ㄴㅇㅇ을

8 政

. 50

\%

음홀

马ㄷํ응

.

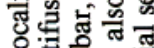
을

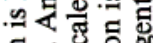

I $\dot{1}$.

잉

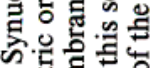
है छㄴㅇㅇ $\infty$ 造

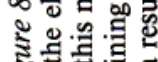




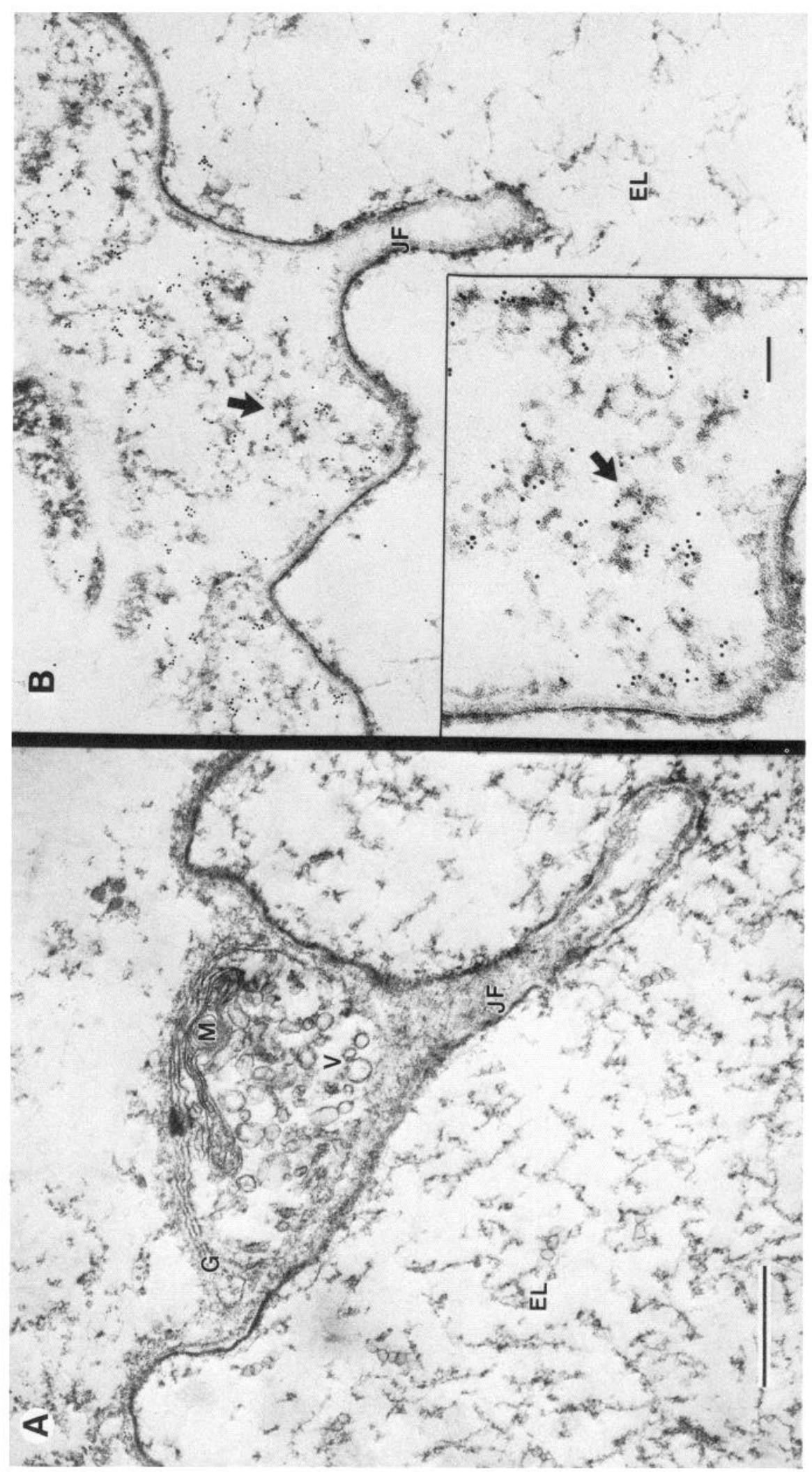

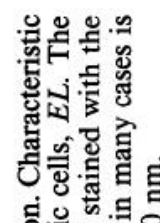

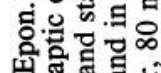

들

5.

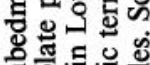
:

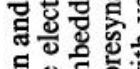
D。

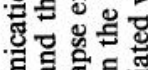
\% 政

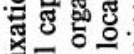
둥 छ.

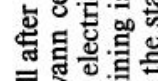

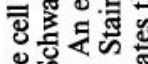
政 동

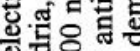

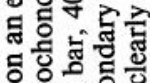

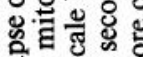
西心

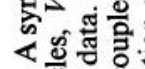
i. 两.

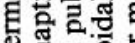
s c5 gis 응응 은 둥 क्षे

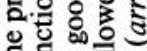

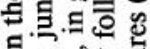

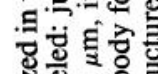
츔율 은 준들

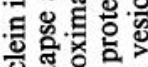
킁믄

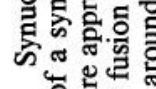

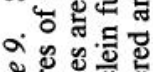

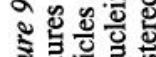

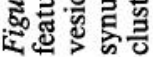


part, for the formation and maintenance of this nuclear localization. A simple explanation of the gradient that begins along the inner lining of the nuclear envelope is an apparent affinity of synuclein for certain membranes. As a result, the affinity constant of synuclein for the membrane will define the slope of the gradient in the direction moving away from the membrane. If the membrane affinity is superimposed upon self-affinity, the large patches of immunoreactivity might be accounted for. Naturally, second- or higher-order interactions could intervene in generating the observed localization. For example, synuclein may bind a membrane protein and have little or no affinity for the membrane itself. Presumably synuclein is synthesized in the cytoplasm, and since no obvious signal sequence is apparent, we do not think the membrane-associated fraction of the protein traverses membrane. The mode of localization in the nucleus could involve a lateral diffusion along the endoplasmic reticulum and outer nuclear membrane (Torrisi et al., 1987) or more conventional transport through nuclear pores; however, the precise molecular mechanism that underlies the compartmentalization is unknown.

The other major site of synuclein immunoreactivity is the presynaptic nerve terminal. Three lines of preliminary evidence suggest synuclein may be associated with synaptic vesicles in the nerve terminal. First, the gene was initially identified in an expression vector screen using a serum antibody generated against purified cholinergic synaptic vesicles. Second, the EM localization of synuclein fusion protein antibody shows a higher concentration of immunoreactivity in the vicinity of vesicles. Finally, preliminary cellular fraction experiments-including purification of vesicles using differential centrifugation, sucrose gradients, and glass bead chromatography - suggest that at least a portion of the protein is membrane associated (data not shown).

\section{Proposed functions of a nuclear and synaptic protein}

Since the protein structure predicted from the analysis of cDNA clones most likely rules out an enzymatic role for synuclein in nerve cells, we are left with the possibility of a structural and/ or regulatory function. The conservation between the divergent species, rat and Torpedo, suggests the repeat structure is crucial for synuclein function. An obvious nuclear function for the proteins may be in the regulation of gene expression, via direct interactions with DNA or chromatin. It is important to note, however, that the structure of synuclein does not fall into any of the known categories of DNA regulatory proteins. In the synapse a large number of functions are possible for synuclein, including trafficking, docking, membrane fusion, and ion translocation.

Synuclein could perform distinct, unrelated functions in spatially distant cellular locations; however, one of the most interesting potential roles of the proteins may be to coordinate nuclear and synaptic events. The weak, yet intriguing, homology to rho and ras, as well as the similar localization of protein kinase $C$ in the presynaptic nerve terminal and in some nuclei, leads to the proposal that synuclein may be involved in signal transduction. If, for instance, synuclein performed a regulatory role either indirectly as a regulatory subunit or more directly, both nuclear and synaptic events could be coordinately modulated. In this way, synuclein could be a molecular monitor of cellular conditions, responding to changes in the physiological state of the cell both in the nucleus and the nerve terminal.

\section{References}

Augustine, G. J., M. P. Charlton, and S. J. Smith (1987) Calcium in synaptic transmitter release. Annu. Rev. Neurosci. 10: 633-693.

Baines, A. J. (1987) Synapsin I and the cytoskeleton. Nature 326: 646.

Berridge, M. (1986) Second messenger dualism in neuromodulation and memory. Nature 323: 294-295.

Buckley, K., and R. B. Kelly (1985) Identification of a transmembrane glycoprotein specific for secretory vesicles of neural and endocrine cells. J. Cell Biol. 100: 1284-1294.

Burnette, W. N. (1981) Western blotting: Electrophoretic transfer of proteins from sodium dodecyl sulfate polyacrylamide gels to unmodified nitrocellulose and radiographic detection with antibody and radioiodinated protein A. Anal. Biochem. 112: 195-203.

Cambier, J. C., M. K. Newell, L. B. Justement, J. C. McGuire, K. L. Leach, and Z. Z. Chen (1987) Ia binding ligands and cAMP stimulate nuclear translocation of PKC in B lymphocytes. Nature 327 ; 629-632.

Carlson, S. S., and R. B. Kelly (1980) An antiserum specific for cholinergic synaptic vesicles from electric organ. J. Cell Biol. 87: 98-103.

Carlson, S. S., and K. B. Kelly (1983) A highly antigenic proteoglycanlike component of cholinergic synaptic vesicles. J. Biol. Chem. 258 . 11082-11091.

Carlson, S. S., J. A. Wagner, and R. B. Kelly (1978) Purification of synaptic vesicles from Elasmobranch electric organ and the use of biophysical criteria to demonstrate purity. Biochemistry 17: 11881198.

Geisow, M. J., U. Fritsche, J. M. Hexham, B. Dash, and T. Johnson (1986) A consensus amino-acid sequence repeat in Torpedo and mammalian $\mathrm{Ca}^{2+}$-dependent membrane-binding proteins. Nature 320 : 636-638.

Gilman, A. G. (1984) G proteins and dual control of adenylate cyclase. Cell 36: 577-579.

Goelet, P., V. F. Castellucci, S. Schacher, and E. R. Kandel (1986) The long and the short of long-term memory: A molecular framework. Nature 323: 415-422.

Hooper, J. E., S. S. Carlson, and R. B. Kelly (1980) Antibodies to synaptic vesicles purified from Narcine electric organ bind a subclass of mammalian nerve terminals. J. Cell Biol. 87: 104-113.

Huttner, W. B., W. Schibler, P. Greengard, and P. DeCamilli (1983) Synapsin I (Protein I), a nerve terminal specific phosphoprotein. III. Its association with synaptic vesicles studied in a highly purified synaptic vesicle preparation. J. Cell Biol. 96: 1374-1388.

Huynh, T. V., R. A. Young, and R. W. Davis (1985) Constructing and screening cDNA libraries in $\lambda \mathrm{gt} 10$ and $\lambda \mathrm{gt} 11$. In DNA Cloning: A Practical Approach, D. M. Glover, ed., pp. 49-78, IRL Press.

Israel, M., N. Morel, B. Lesbats, S. Birman, and R. Manaranche (1986) Purification of a presynaptic membrane protein that mediates a calcium-dependent translocation of acetylcholine. Proc. Natl. Acad. Sci. USA 83: 9226-9230.

Kaczmarek, L. K. (1987) The role of protein kinase C in the regulation of ion channels and neurotransmitter release. TINS 10:30-34.

Kaldany, R. R., J. T. Campanelli, M. Schaeffer, M. Shyamala, and R. H. Scheller (1985) I ow molecular weight proteins of Aplysia neurosecretory cells. Peptides (Suppl. 3) 6: 445-449.

Kreiner, T., W. Sossin, and R. H. Scheller (1986) Localization of Aplysia neurosecretory peptides to multiple populations of dense core vesicles. J. Cell Biol. 102: 769-782.

Luft, J. H. (1958) The fine structure of electric tissue. Exp. Cell Res. (Suppl.) 5: 168-182.

Madaule, P., and R. Axel (1985) A novel ras-related gene family. Cell 41: $31-40$.

Maniatis, I., W. Fritsch, and J. Sambrook (1982) Molecular Cloning: A Laboratory Manual, Cold Spring Harbor Laboratory; Cold Spring Harbor, New York.

Matthew, W. D., L. Tsavaler, and L. F. Reichardt (1981) Identification of a synaptic-vesicle specific membrane protein with a wide distribution in neuronal and neurosecretory tissue. J. Cell Biol. 91: 257_ 269.

McCaffery, C. A, and L. J. DeGennaro (1986) Determination and analysis of the primary structure of the nerve terminal specific phosphoprotein, synapsin I. EMBO J. 5: 3167-3173.

McCormick, F., B. F. Clark, T. F. LaCour, M. Kjeldgaard, L. Norskov- 
Lauritsen, and J. Nyborg (1985) A model for the tertiary structure of $\mathrm{p} 21$, the product of the ras oncogene. Science 230: 78-82.

Michell, B., and C. Kirk (1986) G-protein control of inositol phosphate hydrolysis. Nature 323: 112-113.

Myers, M. W., R. A. Lazzarini, V. M. Lee, W. W. Schlaepfer, and D. L. Nelson (1987) The human mid-size neurofilament subunit: A repeated protein sequence and the relationship of its gene to the intermediate filament gene family. EMBO J. 6: 1617-1626.

Noda, M., H. Takahashi, T. Tanabe, M. Toyasato, Y. Furutani, T. Hirose, M. Asai, S. Inayama, T. Miyata, and S. Numa (1982) Primary structure of $\alpha$-subunit precursor of Torpedo californica acetylcholine receptor deduced from cDNA sequence. Nature 299: 793797.

Ohsawa, K., G. H. C. Dowe, S. J. Morris, and V. P. Whittaker (1979) The lipid and protein content of cholinergic synaptic vesicles from the electric organ of Torpedo marmorata purified to constant composition: Implications for vesicle structure. Brain Res. 161: 447-457.

Reichardt, L. F, and R. B. Kelly (1983) A molecular description of the nerve terminal function. Annu. Rev. Biochem. 52: 871-926.

Schumacher, M., S. Camp, Y. Maulet, M. Newton, K. MacPhee-Quigley, S. S. Taylor, T. Freidmann, and P. Taylor (1986) Primary structure of Torpedo california acetylcholinesterase deduced from its cDNA sequence. Nature 319: 407-409.

Schwartz, J. H., and S. M. Greenberg (1987) Molecular mechanisms for memory: Second-messenger induced modifications of protein kinases in nerve cells. Annu. Rev. Neurosci. 10: 459-476.

Tashiro, T., and H. Stadler (1978) Chemical composition of cholinergic synaptic vesicles from Torpedo marmorala based on improved purification. Eur. J. Biochem. 90: 479-487.

Taussig, R., and R. H. Scheller (1986) The Aplysia FMRFamide gene encodes sequences related to mammalian brain peptides. DNA 5, 6: 453-461.

Torrisi, M. R., L. V. Lotti, A. Pavan, G. Migliaccio, and S. Bonatti (1987) Free diffusion to and from the inner nuclear membrane of newly synthesized plasma membrane glycoproteins. J. Cell Biol. 104: 733-737.

Vanen, I., P. Ekblom, and P. Laurila (1980) Subcellular compartmentalization of saccharide moieties in cultured normal and malignant cells. J. Cell Biol. 85: 429-434.

Wagner, J. A., S. S. Carlson, and R. B. Kelly (1978) Chemical and physical characterisation of cholinergic synaptic vesicles. Biochemistry $17: 1199-1206$.

Wiedenmann, B., and W. W. Franke (1985) Identification and localization of synaptophysin, an integral membrane glycoprotein of MW 38000 characteristic of presynaptic vesicles. Cell 41: 1017-1028.

Wood, J. G., P. R. Girard, G. J. Mazzei, and J. F. Kuo (1986) Immunocytochemical localization of protein kinase $C$ in identified neuronal compartments of rat brain. J. Neurosci. 6: 2571-2577. 